\title{
Hausdorff Dimension of Order Preserving Sets
}

\author{
J. J. P. Veerman * \\ Rockefeller University, Box 75, New York, NY 10021, USA
}

\begin{abstract}
Let $g$ a $C^{2}$ generic bimodal map of the circle. We prove that the closure of the union of the order preserving recurrent sets with irrational rotation number has Hausdorff dimension zero. This set contains order preserving periodic orbits with each rotation number $p / q$ in the rotation interval of $g$.
\end{abstract}

\section{Introduction and Definitions}

Consider a $C^{2}$ bimodal map $g$ of the circle to itself. We choose $g$ such that the boundaries of the rotation interval $\varrho(g)$ of $g$ are rational. It is easy to see that the requirement holds for $C^{2}$-generic $g$. Note that if one replaces $g$ with $g_{\lambda}=g+\lambda$, with $\lambda \in R$, then the latter requirement holds for Lebesgue almost every $\lambda$ (see Swiatek 1987). Without loss of generality we may choose $g$ such that $g^{\prime}(0)<0$.

Invariant order preserving sets can be assigned a rotation number. Consider the union $\Omega$ of all order preserving minimal sets that have irrational rotation number. One can show (Veerman 1989) that each such set is the unique recurrent set of $f_{t}$, where $f_{t}$ is defined as follows:

- $f_{t}$ is non-decreasing and orientation preserving,

- for $x \in U_{t}$ ("flat spot"), $U_{t}$ an open interval, $f_{t}(x)=t$,

- for $x \notin U_{t}, f_{t}(x)=g(x)$,

- the rotation number of $f_{t}, \varrho(t)$ is irrational.

The set of parameters $t$ for which $\varrho(t)$ is irrational will be called $A$. Let $\Omega_{t}$ be the recurrent set of $f_{t}$. The previous statements can be summarized as

$$
\Omega=\bigcup_{t \in A} \Omega_{t} .
$$

It has been established that the Hausdorff dimension of $\bar{A}, H D(\bar{A})$, is zero (Veerman 1989). In this note, it is our aim to show:

Theorem 1.1. $H D(\bar{\Omega})=0$.

^ Current address: Institute for the Mathematical Science, SUNY at Stony Brook, NY 11794, USA 
We give a brief description of the set $\bar{\Omega}$ (see Veerman 1986). For $t \in A, \Omega_{t}$ is the unique minimal recurrent Cantor set of $f_{t}$. The closure of their union, $\bar{\Omega}$, is again a Cantor set. Each $x$ in $\partial \bar{\Omega}$ lies on an orbit of $f_{t}$ with $t \in \partial \bar{A}$. There are two possible types:

- Order preserving periodic orbits with rotation number $p / q \in \varrho(g)$, and

- receding or advancing order preserving orbits with rotation number $p / q \in \varrho(g)$.

These latter orbits are not recurrent and have as their $\alpha$-and $\omega$-limit set an orbit of the first type. The difference between receding and advancing is that for a receding orbit $F^{q}(x)<x+p$, whereas for an advancing orbit the opposite inequality holds.

It is clear, then, that $\partial \bar{\Omega}$ contains only countably many points. The previous theorem is implied by:

Proposition 1.2. $H D(\Omega)=0$.

Finally, we remark (Veerman 1987) that for each irrational $\varrho$, there is a neighborhood $n$ of $\varrho$, such that $g$ has a unique order preserving unstable orbit with rotation number $p / q \in n$. This orbit has unique receding and advancing order preserving homoclinic orbits. For $p / q \in n$, these are precisely the orbits that lie in $\partial \bar{\Omega}$.

\section{Expansivity}

Let $\tau$ be such that $\varrho(\tau)$ is irrational. In this section, we prove that $g^{m}$ restricted to some open neighborhood of $\Omega_{\tau}$ can be extended to $h$, an expanding $C^{2}$ map of degree $m$. For a definition of the Hausdorff distance, we refer to Falconer (1985).

From now on, we will denote the closed set $S^{1}-\bigcup_{j=0}^{m-1} f_{t}^{-j}\left(U_{t}\right)$ by $E_{m}(t)$. Note that these sets have a partial ordering given by $\ldots E_{n+1}(t) \subset E_{n}(t) \ldots \subset E_{0}(t)$. We call $f_{t}(\gamma, m)$ expansive if:

- there exist $m \geqq 1$ and $\gamma>1$ such that for all $x \in E_{m}(\tau)$ we have:

$$
D f_{\tau}^{m}(x) \geqq \gamma>1 \text {. }
$$

We will call $f_{t}$ uniform $(\gamma, m)$ expansive if:

- for each $\tau \in A$, there is an $\varepsilon>0$ such that for each $t$ in an $\varepsilon$-neighborhood $T_{\varepsilon}$ of $\tau, f_{t}$ is $(\gamma, m)$ expansive.

We have:

Proposition 2.1 (Veerman 1989). There exist $\gamma>1$ and $m>0$ such that $f_{t}$ uniform $(\gamma, m)$ expansive.

Proposition 2.2. There is an open neighborhood $N$ of $\Omega_{\tau}$ such that $\left.\mathrm{g}^{m}\right|_{N}$ can be extended to an expanding map $h$ which is $C^{2}$ and has degree $m+1$.

Proof. Let $N=\bigcup_{t \in T_{\varepsilon}} E_{m}(t)$. According to Proposition 2.1, we may choose $\varepsilon$ so small that $f_{t}$ is $(\gamma, m)$ expansive for all $t \in T_{\varepsilon}$. At $t=\tau$, the rotation number of $f_{\tau}$ is irrational 


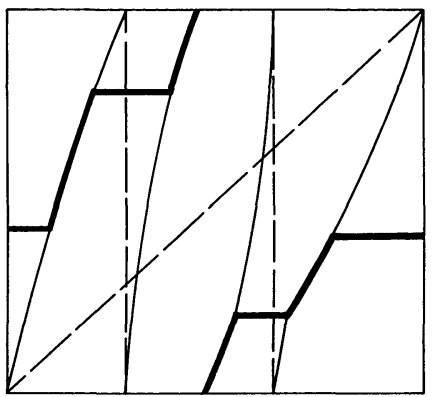

Fig. 2.1. The construction of the extension $h$ of $\left.g^{m}\right|_{N}$

and so the inverse images of $U_{\tau}$ do not intersect or touch $U_{\tau}$. Therefore, $t \rightarrow E_{m}(t)$ is continuous in the Hausdorff topology at $t=\tau$. So, for $\varepsilon$ small enough, $N$ will consist of $m+1$ intervals on which $g^{m}$ is expanding. By restricting $\varepsilon$ again, we can make sure that the Hausdorff distance between the graph of $\left.g^{m}\right|_{J}$ and the diagonal is positive. Then we can extend $\left.g^{m}\right|_{N}$ to $h$ (see Fig. 2.1), where $h$ has the same smoothness as $f$ and has degree $m+1$.

\section{Symbolic Dynamics}

We set up the symbolic dynamics for $h$ needed for the proof of the main result. Lebesgue measure is denoted by $\mu$.

Denote by $I(0)$ and $I(1)$ the intervals $[0, \alpha)$ and $[\alpha, 1)$, where $\alpha$ is the inverse of 0 under $h$. Let $I\left(i_{0} i_{1} \ldots i_{n}\right)$ be defined by:

$$
x \in I\left(i_{0} i_{1} \ldots i_{n}\right) \Leftrightarrow g^{r}(x) \in I\left(i_{r}\right) .
$$

The sequence $\left\{i_{r}\right\}_{r=0}^{\infty}$ will be referred to as the binary expansion of $x$.

According to Veerman (1987) every point with order preserving orbit under $g$ such that its orbit has rotation number $\varrho$ has a binary expansion $\left\{i_{r}\right\}_{r=0}^{\infty}$ that can be constructed as follows (compare Fig. 3.1): There is a $d$ such that

$$
i_{k}=\operatorname{int}\{k \varrho+d\}-\operatorname{int}\{(k-1) \varrho+d\},
$$

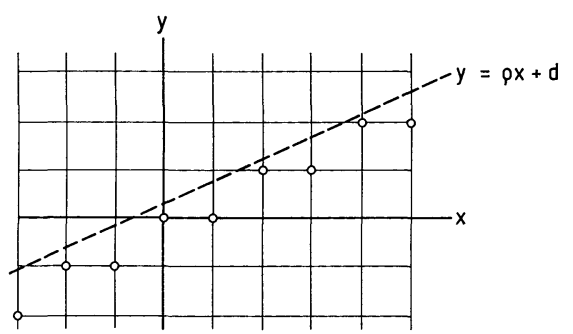

Fig. 3.1. The construction of the binary expansion of a point whose orbit is order preserving 
or

$$
i_{k}=\operatorname{int}^{*}\{k \varrho+d\}-\text { int }^{*}\{(k-1) \varrho+d\},
$$

where

$$
\text { int }^{*}\{x\}=\left\{\begin{array}{ll}
x & \text { if } x \text { is integer valued } \\
\text { int }\{x\}+1 & \text { else }
\end{array} .\right.
$$

In the next section, we need to reason with expanding maps. So, we translate the above statements to the language appropriate for a degree $m+1$ map $h$.

Let $j_{r} \in\{0, \ldots, m+1\}$ and, as before, $J\left(j_{0}\right)$ are the successive inverse images of $[0,1)$. The sequence $\left\{j_{r}\right\}_{r=0}^{\infty}$ associated with a point $x$ iterated under $h$ is called the $m+1$ expansion of $x$. For each order preserving orbit of $g$ contained in $N$, we can construct its binary expansion and its $m+1$ expansion. Recall from the previous section that $g^{m}$ coincides with $h$ on the open set $N$.

Lemma 3.1 ("bounded geometry"). There are constants $1<\alpha \leqq m+1$ and $\beta \geqq m+1$ such that for all $n, i_{n}$ :

$$
\alpha<\frac{\mu\left(J\left(j_{1} \ldots j_{n-1}\right)\right)}{\mu\left(J\left(j_{0} j_{1} \ldots j_{n-1}\right)\right)}<\beta .
$$

Proof. We have $h\left(J\left(j_{0} j_{1} \ldots j_{n-1}\right)\right)=J\left(j_{1} \ldots j_{n-1}\right)$. So the ratio of the lemma equals $h^{\prime}(x)$ for some $x$. But $h$, by Proposition 2.2 , is $C^{2}$, expanding, and of degree $m+1$.

\section{The Hausdorff Dimension of Order Preserving Sets}

In this section, we prove the main result. For the definition of Hausdorff $s$-measure and Hausdorff dimension, we refer to Falconer (1985).

Partition the circle into $N=(m+1)^{n}$ intervals $J\left(j_{0} \ldots j_{n-1}\right)$ as defined in the previous section. Let $S$ be an invariant set such that $S=S \cap \bigcup_{\left\{j_{r}\right\} \in K} J\left(j_{0} \ldots j_{n-1}\right)$, where the cardinality of $K$ is $N_{S}$. So, orbits in $S$ can only land in $N_{S}$ of the $N$ intervals.

Lemma 4.1. $H D(S) \leqq \frac{1}{n} \frac{\ln N_{S}}{\ln \alpha}$.

Proof. Let $\Gamma_{Q}$ be the space of coverings $\left\{\Delta_{i}\right\}_{i=1}^{\infty}$ of $S$ with diameter, at most, $\alpha^{-Q n}$. Denote the Hausdorff $s$-measure by $H_{s}(S)$. Then, by Lemma 3.1, we can cover $S$ with a member of $\Gamma_{Q}$ that has at most $N_{S}^{1+\operatorname{int}\{Q\}}$ intervals. So,

$$
H_{s}(S)=\lim _{Q \rightarrow \infty} \inf _{Q} \sum_{i=0}^{\infty}\left[\mu\left(\Delta_{i}\right)\right]^{s} \leqq \lim _{Q \rightarrow \infty} N_{S}^{1+\operatorname{int}\{Q\}} \alpha^{-Q n s} .
$$

This is equal to zero if

$$
s>\frac{1}{n} \frac{\ln N_{s}}{\ln \alpha} .
$$

Let $S(n)$ be the (invariant) set of points in $N$ with binary expansion such that: 
- All finite subsequences of length $n$ or less are order preserving.

Note that ...S(3) $\subset S(2) \subset S(1)$.

Proposition 4.2. $H D(S(n)) \leqq \frac{1}{n} \frac{\ln 2 n^{4}}{\ln \alpha}$.

Proof. According to the previous lemma, we only have to prove that $N_{S(n)} \leqq 2 n^{4}$. Note that from (3.1) and (3.2) follows that the shift

$$
\sigma: i_{j} \rightarrow i_{j-1}, \quad j>0,
$$

corresponds to

$$
d \rightarrow\{d+\varrho\} \bmod 1
$$

Therefore, the previous statement is implied if the total number of solutions to (3.1) and (3.2) restricted to $k \in\{0, \ldots, n-1\}$ is at most $2 n^{4}$.

Write these solutions as $s_{n}(\varrho, d)$. For a given solution, we can change $d$ and $\varrho$ in the construction without changing $s_{n}(\varrho, d)$, as long as $\{\varrho k+d\} \bmod 1 \neq 0$ for $k \in\{0, \ldots, n-1\}$. So the number of solutions to (3.1) is at most equal to the number of different lines $\varrho x+d$ intersecting precisely two distinct lattice points $\left(l_{1}, l_{2}\right)$ and $\left(l_{3}, l_{4}\right)$, where $l_{i} \in\{0, \ldots, n-1\}$. This number is less than $n^{4}$. The same must be done to count the solutions to (3.2).

As we noted before, the set of all order preserving orbits under $g$ which are contained in $N$, is equal to the set of points whose binary expansion is generated according to (3.1) and (3.2) for $\varrho$ in some interval. So for all $n$, we have the inclusion:

$$
S \subset S(n) \Rightarrow H D(S) \leqq H D(S(n)) .
$$

Corollary 4.3. $H D(\Omega \cap N)=0$.

Proof. The set $S$ we defined above contains $\Omega \cap N$.

Since we can cover $\Omega$ with a countable number of open sets $N_{i}$, Proposition 1.2, and therefore the main result, Theorem 1.1, follows immediately.

Acknowledgements. This work was supported by DOE grant DE-AC-02-83-ER-13044.

\section{References}

Falconer, K.J.: The geometry of fractal sets. Cambridge: Cambridge University Press 1985 Veerman, J.J.P.: On resonance widths in dynamical systems. Thesis, Cornell University 1986 Veerman, J.J.P.: Phys. 29 D, 191-201 (1987)

Veerman, J.J.P.: Irrational rotation numbers. Preprint 1987. Nonl. 2, 419-428 (1989)

Smale, S.: Bull. Am. Math. Soc. 73, 747-817 (1967)

Swiatek, G.: Endpoints of rotation intervals for maps of the circle. Preprint Warsaw 1987

Communicated by J. N. Mather

Received October 1, 1988 
